\title{
Global existence of solutions for 1-D nonlinear wave equation of sixth order at high initial energy level
}

\author{
Jihong Shen ${ }^{2}$, Yanbing Yang ${ }^{1}$ and Runzhang $\mathrm{Xu}^{1,2^{*}}$
}

\section{"Correspondence: \\ xurunzh@163.com \\ ${ }^{1}$ College of Automation, Harbin \\ Engineering University, Harbin, \\ 150001, People's Republic of China \\ ${ }^{2}$ College of Science, Harbin \\ Engineering University, Harbin, \\ 150001, People's Republic of China}

\begin{abstract}
This paper considers the Cauchy problem of solutions for a class of sixth order 1-D nonlinear wave equations at high initial energy level. By introducing a new stable set we derive the result that certain solutions with arbitrarily positive initial energy exist globally.
\end{abstract}

Keywords: Cauchy problem; sixth order wave equation; global existence; arbitrarily positive initial energy; potential well

\section{Introduction}

In this paper, we consider the Cauchy problem for the following 1-D nonlinear wave equation of sixth order:

$$
\begin{aligned}
& u_{t t}-a u_{x x}+u_{x x x x}+u_{x x x x t t}=f\left(u_{x}\right)_{x}, \quad(x, t) \in \mathbb{R} \times(0, \infty), \\
& u(x, 0)=u_{0}(x), \quad u_{t}(x, 0)=u_{1}(x), \quad x \in \mathbb{R},
\end{aligned}
$$

where $f(u)=b|u|^{p}, b>0$ and $p>1$ are constants, $u_{0}(x)$ and $u_{1}(x)$ are given initial data, and $a>0$ is a given constant satisfying certain conditions to be specified later.

When Rosenau [1] was concerned with the problem of how to describe the dynamics of a dense lattice, he discovered Equation (1.1) by a continuum method. Meanwhile onedimensional homogeneous lattice wave propagation phenomena can also be described by Equation (1.1). Since then the well-posedness of Equation (1.1) have been considered by many authors, we refer the reader to [2-4] and the references therein.

Recently, the authors in [5] first considered the Cauchy problem for Equation (1.1). By the contraction mapping principle, they proved the existence and the uniqueness of the local solution for the Cauchy problem of Equation (1.1). By means of the potential well method, they discussed the existence and nonexistence of global solutions to this problem at the sub-critical and critical initial energy level $E(0) \leq d$. So it is natural for us to ask what the weak solution for problem (1.1)-(1.2) behaves at sup-critical initial energy level $E(0)>0$. In this paper we intend to extend the existence of global solutions in [5] with arbitrarily positive initial energy. By using the potential well method [6-9] and introducing a new stable set we show that if the initial data satisfy some conditions, then the corresponding local weak solution with arbitrarily positive initial energy exists globally.

o2014 Shen et al.: licensee Springer. This is an Open Access article distributed under the terms of the Creative Commons Attribution License (http://creativecommons.org/licenses/by/2.0), which permits unrestricted use, distribution, and reproduction in any medium, provided the original work is properly cited. 


\section{Some assumptions and preliminary lemmas}

In this section we give some assumptions and preliminary results to state the main results of this paper. Throughout the present paper, just for simplicity, we denote $L^{p}(\mathbb{R})$ and $H^{s}(\mathbb{R})$ by $L^{p}$ and $H^{s}$, respectively, with the norm $\|\cdot\|_{p}=\|\cdot\|_{L^{p}(\mathbb{R})},\|\cdot\|_{2}=\|\cdot\|_{L^{2}(\mathbb{R})}$ and the inner product $(u, v)=\int_{\mathbb{R}} u v d x$.

For the Cauchy problem (1.1), (1.2) we introduce the energy functional

$$
E(t)=\frac{1}{2}\left(\left\|u_{t}\right\|^{2}+a\left\|u_{x}\right\|^{2}+\left\|u_{x x}\right\|^{2}+\left\|u_{x x t}\right\|^{2}\right)+\frac{b}{p+1} \int_{\mathbb{R}}\left|u_{x}\right|^{p} u_{x} d x
$$

and the Nehari functional

$$
I(t) \equiv I(u)=a\left\|u_{x}\right\|^{2}+\left\|u_{x x}\right\|^{2}+b \int_{\mathbb{R}}\left|u_{x}\right|^{p} u_{x} d x
$$

Moreover we define a new stable set, which will be used to obtain the existence of a global solution with arbitrarily positive initial energy,

$$
\mathcal{W}=\left\{u \in H^{2}(\mathbb{R}) \mid I(u)>\left\|u_{t}\right\|^{2}+\left\|u_{x x t}\right\|^{2}\right\}
$$

We show the following local existence theorem, which has been given in [5].

Theorem 2.1 [5] Suppose that $\frac{3}{2}<s<p+1, u(x, 0) \in H^{s}, u_{t}(x, 0) \in H^{s}$. Then problem (1.1)(1.2) admits a unique local solution $u(x, t)$ defined on a maximal time interval $\left[0, T_{0}\right)$ with $u(x, t) \in C^{1}\left(H^{s},\left[0, T_{0}\right)\right)$. Moreover if

$$
\sup _{t \in\left[0, T_{0}\right)}\left(\|u(x, t)\|_{H^{s}}+\left\|u_{t}(x, t)\right\|_{H^{s}}\right)<\infty
$$

then $T_{0}=\infty$.

\section{The main result and proof}

In order to help the readers quickly get the main idea of the present paper, we show the main theorem in the beginning of this section.

Theorem 3.1 Let $2 \leq s<p+1, u_{0}(x), u_{1}(x) \in H^{s}$ be given and let (3.1) hold. Assume that $E(0)>0, u_{0} \in \mathcal{W}$ and the initial data satisfy

$$
\left\|u_{0}\right\|^{2}+\left\|u_{0 x x}\right\|^{2}+2\left(u_{0}, u_{1}\right)+2\left(u_{0 x x}, u_{1 x x}\right)+\frac{2(p+1)}{p+3} E(0)<0 .
$$

Then the existence time of a global solution for problem (1.1)-(1.2) is infinite.

In what follows, we show a preliminary lemma about the monotonicity of the functional $\|u(x, t)\|^{2}+\left\|u_{x x}(x, t)\right\|^{2}$, which will be used to prove the invariance of the new stable set $\mathcal{W}$ under the flow of problem (1.1)-(1.2).

Lemma 3.2 Let $u_{0}(x), u_{1}(x) \in H^{2}$ be given and $u(x, t)$ be the solution of Equation (1.1) with initial data $\left(u_{0}, u_{1}\right)$. Assume that $E(0)>0$ and the initial data satisfy Equation (3.1), then the map $\left\{t \mapsto\|u(t)\|^{2}+\left\|u_{x x}(t)\right\|^{2}\right\}$ is strictly decreasing as long as $u(x, t) \in \mathcal{W}$. 
Proof Let

$$
F(t)=\|u(t)\|^{2}+\left\|u_{x x}(t)\right\|^{2}
$$

then we get

$$
F^{\prime}(t)=2\left(u, u_{t}\right)+2\left(u_{x x}, u_{x x t}\right)
$$

and

$$
F^{\prime \prime}(t)=2\left(u, u_{t t}\right)+2\left\|u_{t}\right\|^{2}+2\left(u_{x x}, u_{x x t t}\right)+2\left\|u_{x x t}\right\|^{2} .
$$

Note that by testing Equation (1.1) with $u$ we have

$$
\left(u_{t t}, u\right)+\left(u_{x x x x t t}, u\right)+a\left\|u_{x}\right\|^{2}+\left\|u_{x x}\right\|^{2}=-\left(f\left(u_{x}\right), u_{x}\right) \quad \text { for } t \in[0, \infty) .
$$

Then, Equation (3.4) becomes

$$
F^{\prime \prime}(t)=2\left\|u_{t}\right\|^{2}+2\left\|u_{x x t}\right\|^{2}-2 I(u) .
$$

Furthermore, from $u(t) \in \mathcal{W}$ we have $F^{\prime \prime}(t)<0$ for $t \in\left[0, T_{\max }\right)$. Obviously from $E(0)>0$ and (3.1) we can get

$$
2 \int_{\mathbb{R}} u_{0} u_{1} d x+2 \int_{\mathbb{R}} u_{0 x x} u_{1 x x} d x<0,
$$

which implies $F^{\prime}(0)=\left(u_{0}, u_{1}\right)+\left(u_{0 x x}, u_{1 x x}\right)<0$. It is easy to see that $F^{\prime}(t)<F^{\prime}(0)<0$, namely, $F^{\prime}(t)<0$. Therefore, we find that the map $\left\{t \mapsto\|u(t)\|^{2}+\left\|u_{x x}(t)\right\|^{2}\right\}$ is strictly decreasing.

Subsequently we show the invariance of the new stable set $\mathcal{W}$ under the flow of problem (1.1)-(1.2), which plays a key role in proving existence of global solutions for problem (1.1)(1.2) at high initial energy level $E(0)>0$.

Lemma 3.3 (Invariant set) Let $u_{0}(x), u_{1}(x) \in H^{2}$ be given and $u(x, t)$ be a weak solution of problem (1.1)-(1.2) with maximal existence time interval [0, $\left.T_{0}\right), T_{0} \leq+\infty$. Assume that the initial data satisfy (3.1). Then all solutions of problem (1.1)-(1.2) with $E(0)>0$ belong to $\mathcal{W}$, provided $u_{0} \in \mathcal{W}$.

Proof We prove $u(t) \in \mathcal{W}$. If it is false, let $t_{0} \in\left(0, T_{0}\right)$ be the first time that

$$
I\left(u\left(t_{0}\right)\right)=\left\|u_{t}\left(t_{0}\right)\right\|^{2}+\left\|u_{x x t}\left(t_{0}\right)\right\|^{2},
$$

namely,

$$
I(u(t))>\left\|u_{t}\right\|^{2}+\left\|u_{x x t}\right\|^{2} \quad \text { for } t \in\left[0, t_{0}\right) .
$$


Let $F(t)$ be defined as (3.2) above. Hence by Lemma 3.2, we see that $F(t)$ and $F^{\prime}(t)$ are strictly decreasing on the interval $\left[0, t_{0}\right)$. And then by $(3.1)$, for all $t \in\left(0, t_{0}\right)$, we have

$$
F(t)<\left\|u_{0}\right\|^{2}+\left\|u_{0 x x}\right\|^{2}<-2\left(u_{0}, u_{1}\right)-2\left(u_{0 x x}, u_{1 x x}\right)-\frac{2(p+1)}{p+3} E(0) .
$$

Therefore from the continuity of $\|u(t)\|^{2}+\left\|u_{x x}(t)\right\|^{2}$ in $t$ we get

$$
F\left(t_{0}\right)<\left\|u_{0}\right\|^{2}+\left\|u_{0 x x}\right\|^{2}<-2\left(u_{0}, u_{1}\right)-2\left(u_{0 x x}, u_{1 x x}\right)-\frac{2(p+1)}{p+3} E(0) .
$$

On the other hand, by (2.1) and (2.2) we can obtain

$$
\begin{aligned}
E(0)= & E\left(t_{0}\right)=\frac{1}{2}\left(\left\|u_{t}\left(t_{0}\right)\right\|^{2}+a\left\|u_{x}\left(t_{0}\right)\right\|^{2}+\left\|u_{x x}\left(t_{0}\right)\right\|^{2}+\left\|u_{x x t}\left(t_{0}\right)\right\|^{2}\right) \\
& +\frac{b}{p+1} \int_{\mathbb{R}}\left|u_{x}\left(t_{0}\right)\right|^{p} u_{x}\left(t_{0}\right) d x \\
= & \frac{1}{2}\left(\left\|u_{t}\left(t_{0}\right)\right\|^{2}+\left\|u_{x x t}\left(t_{0}\right)\right\|^{2}\right)+\frac{1}{p+1} I\left(u\left(t_{0}\right)\right) \\
& +\left(\frac{1}{2}-\frac{1}{p+1}\right)\left(a\left\|u_{x}\left(t_{0}\right)\right\|^{2}+\left\|u_{x x}\left(t_{0}\right)\right\|^{2}\right) .
\end{aligned}
$$

Recalling (3.6) we have

$$
\begin{aligned}
E(0)= & \left(\frac{1}{2}+\frac{1}{p+1}\right)\left(\left\|u_{t}\left(t_{0}\right)\right\|^{2}+\left\|u_{x x t}\left(t_{0}\right)\right\|^{2}\right) \\
& +\left(\frac{1}{2}-\frac{1}{p+1}\right)\left(a\left\|u_{x}\left(t_{0}\right)\right\|^{2}+\left\|u_{x x}\left(t_{0}\right)\right\|^{2}\right) \\
\geq & \frac{p+3}{2(p+1)}\left(\left\|u_{t}\left(t_{0}\right)\right\|^{2}+\left\|u_{x x t}\left(t_{0}\right)\right\|^{2}\right) .
\end{aligned}
$$

Then from the following equalities:

$$
\begin{aligned}
& \left\|u_{t}\left(t_{0}\right)\right\|^{2}=\left\|u_{t}\left(t_{0}\right)+u\left(t_{0}\right)\right\|^{2}-\left\|u\left(t_{0}\right)\right\|^{2}-2\left(u\left(t_{0}\right), u_{t}\left(t_{0}\right)\right), \\
& \left\|u_{x x t}\left(t_{0}\right)\right\|^{2}=\left\|u_{x x t}\left(t_{0}\right)+u_{x x}\left(t_{0}\right)\right\|^{2}-\left\|u_{x x}\left(t_{0}\right)\right\|^{2}-2\left(u_{x x}\left(t_{0}\right), u_{x x t}\left(t_{0}\right)\right)
\end{aligned}
$$

and (3.8), we can derive

$$
\begin{aligned}
E(0) \geq & A\left\|u_{t}\left(t_{0}\right)+u\left(t_{0}\right)\right\|^{2}+A\left\|u_{x x t}\left(t_{0}\right)+u_{x x}\left(t_{0}\right)\right\|^{2}-A\left\|u\left(t_{0}\right)\right\|^{2} \\
& -A\left\|u_{x x}\left(t_{0}\right)\right\|^{2}-2 A\left(u\left(t_{0}\right), u_{t}\left(t_{0}\right)\right)-2 A\left(u_{x x}\left(t_{0}\right), u_{x x t}\left(t_{0}\right)\right) \\
\geq & -A\left(\left\|u\left(t_{0}\right)\right\|^{2}+\left\|u_{x x}\left(t_{0}\right)\right\|^{2}\right)-2 A\left(\left(u_{0}, u_{1}\right)+\left(u_{0 x x}, u_{1 x x}\right)\right),
\end{aligned}
$$

where $A=\frac{p+3}{2(p+1)}$. Or equivalently

$$
F\left(t_{0}\right) \geq-2\left(u_{0}, u_{1}\right)-2\left(u_{0 x x}, u_{1 x x}\right)-\frac{2(p+1)}{p+3} E(0),
$$

which contradicts the first inequality of (3.7). This completes the proof. 
At this point we can prove the global existence for the solution of problem (1.1)-(1.2) with arbitrarily positive initial energy.

Proof of Theorem 3.1 From Theorem 2.1 there exists a unique local solution of problem (1.1)-(1.2) defined on a maximal time interval $\left[0, T_{0}\right)$. Let $u(t)$ be the weak solution of problem (1.1)-(1.2) with $E(0)>0, u_{0} \in \mathcal{W}$ and (3.1). Then from Lemma 3.3 we have $u(x, t) \in \mathcal{W}$, namely,

$$
I(u(t))>\left\|u_{t}\right\|^{2}+\left\|u_{x x t}\right\|^{2} \quad \text { for } t \in\left[0, T_{0}\right) .
$$

Therefore from (2.1)-(2.2) and (3.6), we can obtain

$$
\begin{aligned}
E(0) & =E(t)=\frac{1}{2}\left(\left\|u_{t}\right\|^{2}+a\left\|u_{x}\right\|^{2}+\left\|u_{x x}\right\|^{2}+\left\|u_{x x t}\right\|^{2}\right)+\frac{b}{p+1} \int_{\mathbb{R}}\left|u_{x}\right|^{p} u_{x} d x \\
& =\frac{1}{2}\left(\left\|u_{t}\right\|^{2}+\left\|u_{x x t}\right\|^{2}\right)+\frac{p-1}{2(p+1)}\left(a\left\|u_{x}\right\|^{2}+\left\|u_{x x}\right\|^{2}\right)+\frac{1}{p+1} I(u) \\
& >\frac{p+3}{2(p+1)}\left(\left\|u_{t}\right\|^{2}+\left\|u_{x x t}\right\|^{2}\right)+\frac{p-1}{2(p+1)}\left(a\left\|u_{x}\right\|^{2}+\left\|u_{x x}\right\|^{2}\right),
\end{aligned}
$$

which implies

$$
u(x, t), u_{t}(x, t) \text { are bounded in } C^{1}\left(0, T_{0} ; H^{s}\right) .
$$

Hence from Theorem 2.1 it follows that $T_{0}=\infty$ and the solution of problem (1.1)-(1.2) exists globally. This completes the proof of Theorem 3.1.

\section{Remarks}

As we all know, the global existence and finite time blow-up results of solutions for problem (1.1)-(1.2) at the sub-critical and critical initial energy level $E(0) \leq d$ were obtained in [5], where $E(0)$ is the total initial energy and $d$ is the depth of the introduced potential well. The present paper derives some sufficient conditions on the initial data such that the solution exists globally at the sup-critical initial energy level $E(0)>0$. However, to the best of our knowledge there is no result as regards the blow-up result of solutions with arbitrary positive initial energy for the Cauchy problem (1.1)-(1.2); obviously this is an open problem. Indeed we made a try to treat this issue at the same time, however, we did not derive the invariant of an unstable set as a result of the lack of a Poincaré inequality.

Competing interests

The authors declare that they have no competing interests.

\section{Authors' contributions}

The work presented here was carried out in collaboration between all authors. RZ found the motivation of this paper and suggested the outline of the proofs. JH provided many good ideas for completing this paper. YB finished the proof of the main theorem. All authors have contributed to, read and approved the manuscript.

\section{Acknowledgements}

We thank the referees for their valuable suggestions which helped us improve the paper so much. This work was supported by the National Natural Science Foundation of China (11101102), China Postdoctoral Science Foundation (2013M540270), Postdoctoral Science Foundation OF Heilongjiang Province, the Support Plan for the Young College Academic Backbone of Heilongjiang Province (1252G020), Foundational Science Foundation of Harbin Engineering University and Fundamental Research Funds for the Central Universities. 


\section{References}

1. Rosenau, P: Dynamics of dense lattices. Phys. Rev. B 36, 5868-5876 (1987)

2. Wang, SB, Chen, GW: Small amplitude solutions of the generalized imbq equation. J. Math. Anal. Appl. 274, 846-866 (2002)

3. Lin, Q, Wu, YH, Ryan, L: On the Cauchy problem for a generalized Boussinesq equation. J. Math. Anal. Appl. 353 186-195 (2009)

4. Wang, SB, Wu, YH: On the asymptotic behavior of solution for the generalized IBq equation with hydrodynamical damped term. J. Differ. Equ. 252, 4243-4258 (2012)

5. Wang, YZ, Wang, YX: Existence and nonexistence of global solutions for a class of nonlinear wave equations of higher order. Nonlinear Anal. 72, 4500-4507 (2010)

6. Xu, RZ, Yang, YB, Chen, SH, Su, J, Shen, JH, Huang, SB: Nonlinear wave equations and reaction-diffusion equations with several nonlinear source terms of different signs at high energy level. ANZIAM J. 54, 153-170 (2013)

7. Shen, JH, Yang, YB, Chen, SH, Xu, RZ: Finite time blow up of fourth-order wave equations with nonlinear strain and source terms at high energy level. Int. J. Math. 24, 1350043 (2013)

8. Xu, RZ, Yang, YB: Finite time blow-up for the nonlinear fourth-order dispersive-dissipative wave equation at high energy level. Int. J. Math. 23, 1250060 (2012)

9. Xu, RZ, Shu, J: Global existence and finite time blow-up for a class of semilinear pseudo-parabolic equations. J. Funct. Anal. 264, 2732-2763 (2013)

10.1186/1687-2770-2014-31

Cite this article as: Shen et al.: Global existence of solutions for 1-D nonlinear wave equation of sixth order at high initial energy level. Boundary Value Problems 2014, 2014:31

\section{Submit your manuscript to a SpringerOpen ${ }^{\circ}$ journal and benefit from:}

- Convenient online submission

- Rigorous peer review

- Immediate publication on acceptance

- Open access: articles freely available online

- High visibility within the field

- Retaining the copyright to your article 